\title{
A Dynamic Substrate is Required for MhuD-catalyzed Degradation of Heme to Mycobilin
}

\author{
Biswash Thakuri, Bruce D. O’Rourke, Amanda B. Graves, and Matthew D. Liptak* \\ Department of Chemistry, University of Vermont, Burlington, Vermont 05405, United States
}

\begin{abstract}
The non-canonical heme oxygenase MhuD from Mycobacterium tuberculosis binds a heme substrate that adopts a dynamic equilibrium between planar and out-of-plane ruffled conformations. MhuD degrades this substrate to an unusual mycobilin product via successive monooxygenation and dioxygenation reactions. This article establishes a causal relationship between heme substrate dynamics and MhuD-catalyzed heme degradation resulting in a refined enzymatic mechanism. UV/Vis absorption (Abs) and electrospray ionization mass spectrometry (ESI-MS) data demonstrated that a second-sphere substitution favoring population of the ruffled heme conformation changed the rate-limiting step of the reaction resulting in a measurable build-up of the monooxygenated meso-hydroxyheme intermediate. In addition, UV/Vis Abs and ESI-MS data for a second-sphere variant that favored the planar substrate conformation showed that this change altered the enzymatic mechanism resulting in an $\alpha$-biliverdin product. Single-turnover kinetic analyses for three MhuD variants revealed that the rate of heme monooxygenation depends upon the population of the ruffled substrate conformation. These kinetic analyses also revealed that the rate of meso-hydroxyheme dioxygenation by MhuD depends upon the population of the planar substrate conformation. Thus, the ruffled heme conformation supports rapid heme monooxygenation by MhuD, but further oxygenation to the mycobilin product is inhibited. In contrast, the planar substrate conformation exhibits altered heme monooxygenation regiospecificity followed by rapid oxygenation of meso-hydroxyheme. Altogether, these data yielded a refined enzymatic mechanism for MhuD where access to both substrate conformations is needed for rapid incorporation of three oxygen atoms into heme yielding mycobilin.
\end{abstract}

\section{INTRODUCTION}

At physiologically-relevant temperatures, proteins vibrate around their equilibrium structure and these dynamic motions are often essential for their function. Sometimes dynamic motion is dramatic, as is the case for the domain rearrangements of cobalamin-dependent methionine synthase during enzymatic turnover..$^{1-3}$ Other times, more subtle structural changes have important functions as is the case with the allosteric changes to hemoglobin following binding of molecular oxygen..$^{4-6}$ In heme proteins, a source of dynamic motion is out-of-plane deformations of the heme substrate..$^{7-9}$ For clarity, these motions are described based upon the normal coordinates for distortion from a $D_{4 h}$ symmetric tetrapyrrole: waving $\left(e_{g}\right)$, propellering $\left(a_{1 u}\right)$, doming $\left(a_{2 u}\right)$, ruffling $\left(b_{1 u}\right)$, and saddling $\left(b_{2 u}\right)$. A prominent example of a protein that hosts a dynamic heme is Mycobacterium tuberculosis MhuD..$^{10-12}$ MhuD is a noncanonical heme oxygenase $(\mathrm{HO})$ whose heme substrate undergoes a dynamic ruffling deformation, but it has not yet been ascertained whether the substrate dynamics have a functional role.

It has been shown that MhuD binds a dynamic heme and that MhuD degrades heme to a unique product, but a connection between heme ruffling and mycobilin production has been elusive. Magnetic measurements have shown that the large ruffling deformation observed in the X-ray crystal structure of cyanide-inhibited MhuD (MhuD-heme-CN,
PDB ID ${ }_{4} \mathrm{NL}_{5}$ ) arises from a dynamic heme substrate that is present in solution as an equilibrium mixture of planar and ruffled heme..$^{10-12}$ One explanation for the presence of two heme conformations in MhuD is that it is a consequence of the fact that at least four polypeptide conformational states exist. ${ }^{10,13,14}$ Alternatively, from a heme electronic structure viewpoint, the ruffled conformation can be attributed to a hidden pseudo Jahn-Teller distortion arising from vibronic coupling of the ${ }^{2} B_{2 g}$ and ${ }^{2} A_{2 u}$ excited states of the planar species by a $b_{1 u}$ vibration. ${ }^{12,15,16}$ The vibrational frequency for the $b_{1 u}$ out-of-plane ruffling motion is $40-60 \mathrm{~cm}^{-1},{ }^{17,18}$ and thermal energy at $37^{\circ} \mathrm{C}$ is $220 \mathrm{~cm}^{-1}$, so the distortion required for vibronic coupling of the ${ }^{2} B_{2 g}$ and ${ }^{2} A_{2 u}$ excited states is facile. As alluded to above, MhuD is a non-canonical $\mathrm{HO}$ that degrades heme to mycobilin via a meso-hydroxyheme intermediate (Figure 1).19,20 Canonical HOs, which degrade heme to biliverdin, also have a mesohydroxyheme intermediate, suggesting the mechanisms of the two enzymes diverge after the first oxygenation reaction. ${ }^{21}$ These observations suggest that heme ruffling induced by the MhuD active site may be responsible for the novel mycobilin product of this enzyme, but no clear correlation between heme ruffling and $\mathrm{HO}$ degradation product has been reported.

The populations of the planar and ruffled heme conformations in MhuD can be tuned by the $\mathrm{F}_{23} \mathrm{~W}$ and $\mathrm{W} 66 \mathrm{~F}$ substitutions because these residues act like a vice on the 

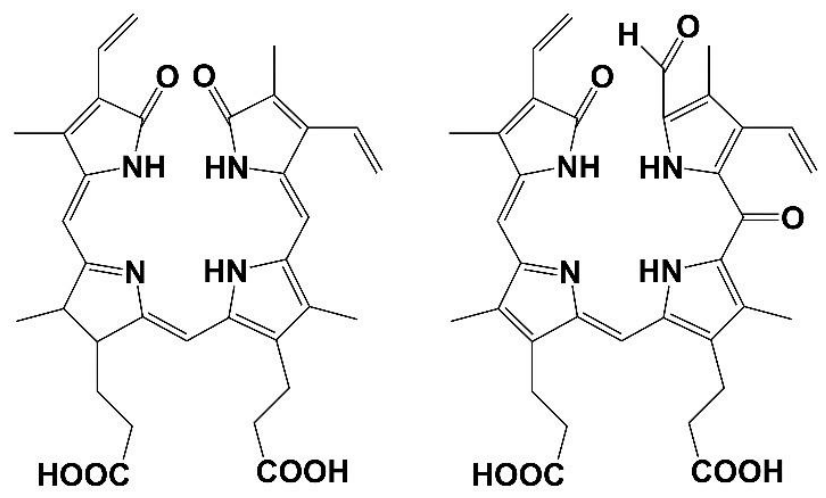

Biliverdin

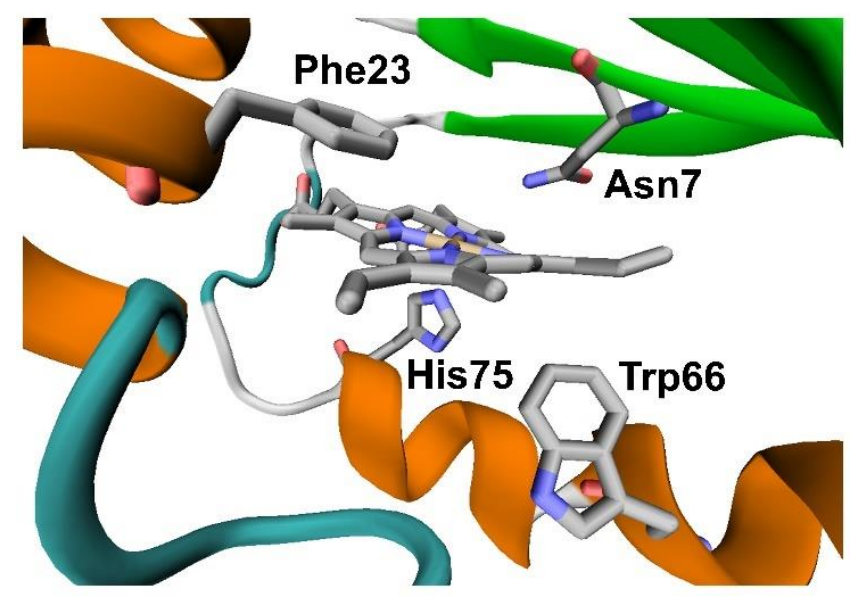

Figure 1. MhuD is a non-canonical heme oxygenase whose heme degradation products and active site structure differ from canonical enzymes (PDB ID $\left.{ }_{4} \mathrm{NL}_{5}\right) \cdot{ }^{10} \mathrm{MhuD}$ produces a trioxygenated mycobilin product, whereas canonical heme oxygenases generate dioxygenated biliverdin (top). His75 is a first-sphere ligand to the heme substrate. Asn7, Phe23, and Trp66 are second-sphere ligands that perturb the heme substrate via non-bonding interactions (bottom).

heme substrate (Figure 1). The $\mathrm{F}_{23} \mathrm{~W}$ and W66F substitutions have been shown to perturb the UV/Vis absorption (Abs) spectrum of MhuD. .0-12 $^{10}$ These spectral changes, along with those observed for W66Y IsdI, ${ }^{22,23}$ have been fully explained by a time-dependent density functional theory model where the only structural perturbation is heme ruffling." The heme electronic ground state, and the energies of the low-lying electronic excited states, in MhuD have also been shown to be perturbed by the $\mathrm{F}_{23} \mathrm{~W}$ and W66F substitutions based upon nuclear magnetic resonance and magnetic circular dichroism characterizations. ${ }^{10-12}$ These electronic structure changes can also be fully explained by pure ruffling deformations, ${ }^{12}$ although the computationally expensive $\mathrm{N}$-electron valence state perturbation theory method must be employed for an adequate description of the strong electron correlation. ${ }^{24-26}$ The $\mathrm{F}_{23} \mathrm{~W}$ and W66F substitutions could also perturb the orientations of the porphyrin side-chains. However, these changes did not need to be invoked to develop accurate computational models of $\mathrm{F}_{23} \mathrm{~W}$ and $\mathrm{W} 66 \mathrm{~F}$ MhuD, ${ }^{11,12}$ nor were these changes observed upon comparison of the X-ray crystal structures of WT and W66Y IsdI (PDB IDs 3QGP and
4FNI).22,23 Thus, the F23W and W66F substitutions are valuable tools that can be used to toggle the ratio of the planar and ruffled heme conformations within the MhuD active site.

To assess the role(s) of a dynamic heme in the MhuD enzyme mechanism, we identified the isomeric products and measured rate constants for MhuD-catalyzed heme oxygenation as a function of substrate conformation. We determined the isomeric products of MhuD-catalyzed heme degradation for enzyme variants that stabilize one of two substrate conformations. ${ }^{12}$ Wild-type (WT) MhuD is known to produce mycobilin, and the R26S variant has been shown to generate predominantly $\alpha$-biliverdin, ${ }^{27}$ but the origin of this difference has yet to be explained. The Michaelis-Menten model is problematic for MhuD-catalyzed heme oxygenation because: MhuD binds the heme substrate tightly with a $K_{\mathrm{d}}$ of $7.6 \mathrm{nM},{ }^{28}$ MhuD-catalyzed heme oxygenation is a multi-step reaction, ${ }^{20}$ and the enzyme is product-inhibited in vitro. ${ }^{14}$ Thus, we have derived single-turnover kinetic expressions that are appropriate for the MhuD-catalyzed reaction in this article. The enzyme catalyzed conversion of heme to meso-hydroxyheme is formally a monooxygenation, ${ }^{20}$ and the influence of ruffling on the rate of this reaction has been assessed using an established assay. ${ }^{13}$ The further oxygenation of meso-hydroxyheme to mycobilin is formally a dioxygenation reaction, ${ }^{19}$ and the rate of this reaction was also assessed here. These experiments have provided important insight into the role of dynamic heme ruffling in the enzymatic mechanism of MhuD.

This article describes a mechanistic study of MhuD-catalyzed heme oxygenation carried out using UV/Vis Abs spectroscopy and mass spectrometry (MS). Forms of MhuD with increased populations of the ruffled and planar substrate conformations were prepared by introducing the $\mathrm{F}_{23} \mathrm{~W}$ and W66F substitutions, ${ }^{11,12}$ respectively. The heme degradation products of WT, $\mathrm{F}_{23} \mathrm{~W}$, and W66F MhuD were identified using UV/Vis Abs spectroscopy and in proteo MS. ${ }^{29}$ In addition, tandem MS was employed to determine the product isomer(s) for each reaction. Next, using a previously described UV/Vis Abs-based assay, ${ }^{13}$ the rates of WT, F23W, and W66F MhuD-catalyzed heme monooxygenation were measured by monitoring the disappearance of the Soret band. Finally, the rate of meso-hydroxyheme oxygenation was quantified by analyzing the time course of a UV/Vis Abs band in the red region of the spectrum. These data have provided significant new insight into the enzymatic mechanism of MhuD, and revealed a new example of protein function derived from protein dynamics.

\section{EXPERIMENTAL DETAILS}

All reagents were purchased from Fisher Scientific and used without further purification unless noted otherwise.

Protein expression and purification. WT, $\mathrm{F}_{23} \mathrm{~W}$, and W66F MhuD were expressed as described previously. Briefly, a pET-22b (Ampr) plasmid encoding WT MhuD was a gift from Prof. Celia Goulding (University of California-Irvine). ${ }^{13}$ The $\mathrm{F}_{23} \mathrm{~W}$ and W66F mutations were introduced using QuikChange site-directed mutagenesis kits 
(Agilent). ${ }^{11,12}$ All three MhuD variants were overexpressed in BL21-GOLD (DE3) cells and grown aerobically in LuriaBertani (LB) medium, as described previously.

WT, F23W, and W66F MhuD were purified in a manner similar to that previously described for $\mathrm{His}_{6}$-tagged WT enzyme. Previous studies have demonstrated that the His ${ }_{6}^{-}$ tag interferes with formation of two heme-bound MhuD, ${ }^{28}$ but this article will exclusively focus on the single hemebound (MhuD-heme) species. WT MhuD was purified using an ÄKTA pure $25 \mathrm{~L}$ fast protein liquid chromatography (FPLC) system. ${ }^{28} \mathrm{~F}_{23} \mathrm{~W}$ MhuD was purified similarly, but a $1.3 \mathrm{mM} / \mathrm{mL}$ elution gradient was employed (Figure $\mathrm{S}_{1}$ ). This procedure yielded $18 \mathrm{mg}$ of $>99 \%$ pure F23W MhuD per liter of LB medium (Figure S2). W66F MhuD was also purified similarly to WT enzyme, but with an elution gradient of $3.3 \mathrm{mM} / \mathrm{mL}$ from 125 to $325 \mathrm{mM}$ imidazole (Figure $\mathrm{S}_{3}$ ). Purification of W66F MhuD yielded $15 \mathrm{mg}$ of $>99 \%$ pure protein per liter of growth medium, as assessed by SDSPAGE (Figure $\mathrm{S}_{4}$ ).

UV/Vis Abs spectroscopy. Heme degradation by MhuD variants was assayed in a manner similar to previously described experiments. WT, F23W, and W66F MhuD-heme were prepared as previously described. $.^{10,13} \mathrm{UV} / \mathrm{Vis}-$ detected heme degradation assays were performed in a manner similar to that previously described, ${ }^{19}$ with several exceptions. Briefly, $50 \mu \mathrm{M}$ MhuD-heme in $50 \mathrm{mM}$ potassium phosphate $\left(\mathrm{KP}_{\mathrm{i}}\right) \mathrm{pH}$ 6.o was reacted with $5 \mathrm{mM}$ ascorbic acid, 10 $\mathrm{mM}$ ethylenediaminetetraacetic acid (EDTA), $840 \mathrm{U} / \mathrm{mL}$ bovine catalase (Sigma-Aldrich), and ${ }_{167} \mathrm{U} / \mathrm{mL}$ bovine superoxide dismutase at $37^{\circ} \mathrm{C}$ while protected from light. For each enzyme variant, UV/Vis Abs spectra were acquired between 700 and $300 \mathrm{~nm}$ with a scan rate of $600 \mathrm{~nm} / \mathrm{min}$, a 1.0 nm data interval, and a o.1 $\mathrm{s}$ integration time using a Cary 100 Bio UV-Vis spectrophotometer at 0, 5, 10, 20, 30, 60 , and 120 min after mixing.

Heme degradation by F23W MhuD was also monitored at a lower temperature with higher time resolution to gain further insight into the reaction catalyzed by this enzyme

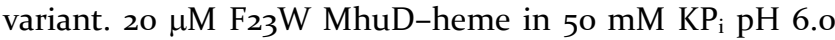
was reacted with $2 \mathrm{mM}$ ascorbic acid, $5 \mathrm{mM}$ EDTA, 840 $\mathrm{U} / \mathrm{mL}$ bovine catalase, and $167 \mathrm{U} / \mathrm{mL}$ bovine superoxide dismutase at room temperature while protected from light. A UV/Vis Abs spectrum of the reaction mixture was acquired every $60 \mathrm{~s}$ for $120 \mathrm{~min}$ using the same instrumental parameters noted above.

Mass spectrometry. Electrospray ionization mass spectrometry (ESI-MS) was used to monitor heme degradation by WT, F23W, and W66F MhuD as a function of time. WT, F23W, and W66F MhuD samples with partially degraded heme were prepared as described above and flash frozen in liquid nitrogen (Airgas) after o, 5, 10, 20, 30, 60, and $120 \mathrm{~min}$ of reaction time. In addition, a control experiment was performed where $50 \mu \mathrm{M}$ hemin chloride in $50 \mathrm{mM} \mathrm{KP}$ i $\mathrm{pH} 6.0$ was reacted with $5 \mathrm{mM}$ ascorbic acid, $10 \mathrm{mM}$ EDTA, 840 $\mathrm{U} / \mathrm{mL}$ bovine catalase (Sigma-Aldrich), and $167 \mathrm{U} / \mathrm{mL}$ bovine superoxide dismutase at $37{ }^{\circ} \mathrm{C}$ for $120 \mathrm{~min}$ before flash freezing the sample in liquid nitrogen. All 22 reactions were loaded onto a $\mathrm{C}_{18}$ column equilibrated with $2 \%$ acetonitrile $(\mathrm{MeCN})$ with $0.1 \%$ formic acid $(v / v)$ in water $(v / v)$, and eluted with a linear gradient from $2 \%$ to $98 \% \mathrm{MeCN}$ with $0.1 \%$ formic acid $(\mathrm{v} / \mathrm{v})$ in water $(v / v)$ over $50 \mathrm{~min}$ using a Shimadzu Prominence high-performance liquid chromatography (HPLC) system. ESI-MS data for $\mathrm{m} / \mathrm{z}$ 6oo-150o was acquired in positive ion mode using an ABI Sciex 4000 QTrap Pro hybrid triple-quadrupole/linear ion trap. Extracted ion chromatograms (EICs) for $\mathrm{m} / \mathrm{z} 583,611$, and 616 target ions were obtained from each total ion chromatogram using BioAnalyst 1.5. Next, each EIC was background subtracted and the integrated areas of each ion as a function of time were determined by integrating under the corresponding chromatographic peak in the background subtracted EIC. The integrated areas of the $\mathrm{m} / \mathrm{z} 583$ and 611 target ions relative to the $\mathrm{m} / \mathrm{z} 616$ target ion at each time point were plotted against time.

Tandem LC-MS experiments were employed to identify the major ions produced by the WT, F23W, and W66F MhuD-catalyzed reactions. Tandem LC-MS data were obtained for the $\mathrm{m} / \mathrm{z} 583,611$ and 616 parent ions of the WT, W66F, and F23W MhuD-catalyzed heme degradation reactions. Evidence for several $\mathrm{m} / \mathrm{z} 583$ isomers was observed, so the 120 minute reactions samples were further separated by liquid chromatography. Up to four isomers were resolved by a linear gradient from $2 \%$ to $82 \%$ MeCN with $0.1 \%$ formic acid $(v / v)$ in water $(v / v)$ over $38 \mathrm{~min}$. All Tandem LC-MS data were acquired using the same instrument described above for ESI-MS. A collision energy of $44 \mathrm{mV}$ was used for fragmentation and the third quadrupole was used as a linear ion trap to scan the fragmentation products of the parent ions.

Kinetic models. Kinetic information was extracted from the UV/Vis Abs data via non-linear least squares fitting to single-turnover kinetic models. The decrease of the MhuD-heme Soret band as a function of time was fit to a pseudo-first order kinetic model:

$A=\left(A_{0}-A_{\infty}\right) e^{-k_{1} t}+A_{\infty}$

where $A_{\mathrm{o}}$ is the absorbance at o $\min , A_{\infty}$ is the absorbance at $\infty \mathrm{min}$, and $k_{1}$ is the rate constant for heme monooxygenation. The decrease of the Soret band Abs intensity versus time was fit to equation 1 using GraphPad Prism 8.o in order to extract $k_{1}$ and its standard error for each MhuD variant.

UV/Vis Abs data were also fit to a single-turnover kinetic model that explicitly considered the contribution of the meso-hydroxyheme intermediate to the spectra:

$A=\left(A_{0}-A_{\infty}\right)\left[e^{-k_{1} t}+\left(\frac{\varepsilon_{m h}}{\varepsilon_{h}}\right)\left(\frac{k_{1}}{k_{2}-k_{1}}\right)\left(e^{-k_{1} t}-e^{-k_{2} t}\right)\right]+A_{\infty}$

where $\varepsilon_{m h}$ is the molar extinction coefficient for meso-hydroxyheme, $\varepsilon_{h}$ is the molar extinction coefficient for heme, and $k_{2}$ is the rate constant for heme dioxygenation. $\varepsilon_{h}$ was determined based upon the pyridine hemochrome assay. ${ }^{30}$ The change in absorbance versus time was fit to equation 2 using GraphPad Prism 8.o to extract $k_{1}, k_{2}, \varepsilon_{m h}$, and their standard errors. Complete derivations of equations 1 and 2 are available in the Supporting Information. 


\section{RESULTS}

The MhuD product depends upon the substrate conformation. Based upon UV/Vis Abs data, WT MhuD degraded heme to mycobilin. As reported previously, two substrate conformations that differ by their degree of outof-plane ruffling are in dynamic equilibrium within the active site of WT enzyme. ${ }^{12}$ Similar to previously reported assays, ${ }^{13,19}$ a reaction was initiated by adding a 10o-fold molar excess of ascorbate to $50 \mu \mathrm{M}$ MhuD-heme in $50 \mathrm{mM} \mathrm{KP_{i }}$ $\mathrm{pH} 6.0$ at $37^{\circ} \mathrm{C}$ and the UV/Vis Abs spectrum of the mixture was monitored versus time (Figure 2).13,19 It should be noted that ascorbate is unlikely to be the physiological electron donor for this reaction, and the redox partner for MhuD has yet to be identified. As was also the case for previous studies that employed cytochrome $\mathrm{P}_{450}$ reductase as the electron donor, ${ }^{13}$ or substituted hydrogen peroxide for both molecular oxygen and the electron donor, ${ }^{20}$ the reaction significantly decreased the Soret band intensity and produced a new UV/Vis Abs band at $540 \mathrm{~nm}$. Based upon the UV/Vis Abs spectrum of mycobilin-bound MhuD, ${ }^{20}$ these observations are consistent with oxygenation of heme to mycobilin by WT enzyme with ascorbate as the electron donor under aerobic conditions. Thus, this study has successfully reproduced past observations regarding the heme degradation reaction catalyzed by WT MhuD, and the role of the two substrate conformations can now be examined.

When the ruffled substrate conformation was favored by the F 23 W substitution, the UV/Vis Abs spectrum of the reaction mixture could be attributed to a build-up of the meso-hydroxyheme intermediate. A previous study demonstrated that the $\mathrm{F}_{23} \mathrm{~W}$ substitution favors population of the ruffled substrate conformation by increasing steric contacts between residue 23 and the porphyrin ring. ${ }^{12}$ The reaction of $\mathrm{F}_{23} \mathrm{~W}$ MhuD with excess ascorbate was monitored by UV/Vis Abs as described above for the WT form of the enzyme, and the formation of a novel $620 \mathrm{~nm}$ band was observed (Figure 2). Based upon the UV/Vis Abs spectrum of meso-hydroxyheme-bound enzyme, we concluded that the UV/Vis Abs band corresponds to a buildup of the meso-hydroxyheme intermediate. ${ }^{20}$ In addition, the UV/Vis Abs spectrum acquired following $2 \mathrm{~h}$ of heme degradation by F23W MhuD has a significantly more intense absorption in the $350-450 \mathrm{~nm}$ region compared to the corresponding data for WT enzyme. This observation is also consistent with formation of meso-hydroxyheme by F23W MhuD since that species retains a Soret band. These data strongly suggest that F23W MhuD has the ability to monooxygenate heme, but subsequent oxygenation reactions may be impaired in this variant.

When the planar substrate conformation was favored by the W66F substitution, an alternate heme degradation product was generated whose UV/Vis Abs spectrum was consistent with biliverdin. The W66F substitution was previously shown to favor population of the planar substrate conformation by reducing a steric clash between residue 66 and the porphyrin ring. ${ }^{11}$ W66F MhuD was assayed under identical conditions to WT enzyme and, surprisingly, production of a broad $680 \mathrm{~nm} \mathrm{UV} / \mathrm{Vis}$ Abs band over the

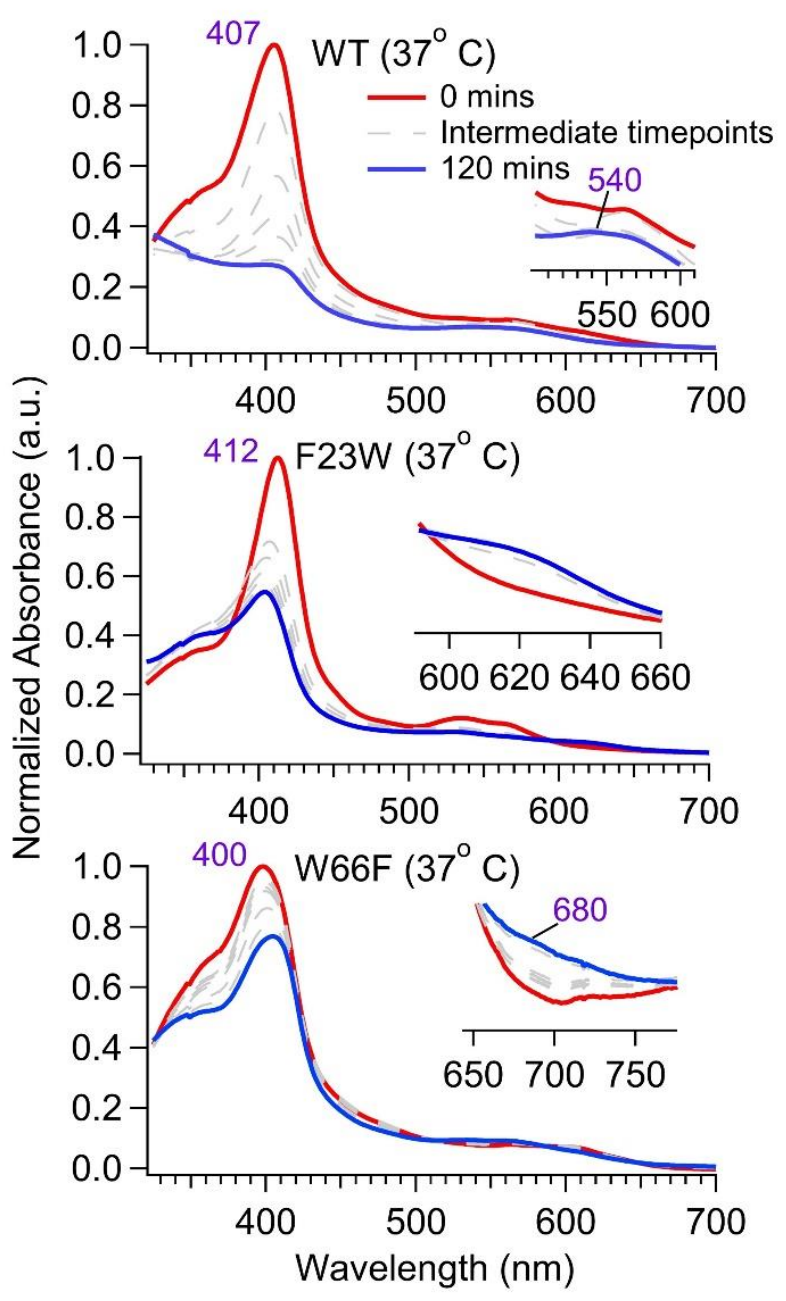

Figure 2. Second-sphere substitutions alter the enzymatic product of MhuD. WT MhuD degrades heme to a new species with a UV/Vis Abs band at $540 \mathrm{~nm}$ (top), which is consistent with production of mycobilin. F23W MhuD-catalyzed heme degradation yields a species with a UV/Vis Abs band at 620 nm (middle), consistent with a build-up of the meso-hydroxyheme intermediate. Heme degradation by W66F MhuD results in a product with a broad UV/Vis Abs band at $680 \mathrm{~nm}$, which suggests biliverdin production. For each variant, the reaction of $50 \mu \mathrm{M}$ MhuD-heme with $5 \mathrm{mM}$ ascorbate in $50 \mathrm{mM}$ $\mathrm{KP}_{\mathrm{i}} \mathrm{pH}$ 6.o was monitored by UV/Vis Abs spectroscopy for $2 \mathrm{~h}$ at $37^{\circ} \mathrm{C}$. Spectral data was acquired o (solid red line), 5, 10, 20, 30, 6o (dashed gray lines), and 120 (solid blue line) mins after mixing.

course of heme degradation reaction was noted (Figure 2). Based upon the UV/Vis Abs spectrum of biliverdin-bound rat $\mathrm{HO},{ }^{31}$ a reasonable assignment for this band is biliverdin. Biliverdin production has been reported previously for R26S MhuD, ${ }^{27}$ but the mechanistic changes that lead to this alternate product remain unknown. The data presented here suggests that the planar substrate conformation may play a role in biliverdin production by MhuD. However, first, the observation that W66F MhuD may degrade heme to biliverdin will be further investigated with MS below. 


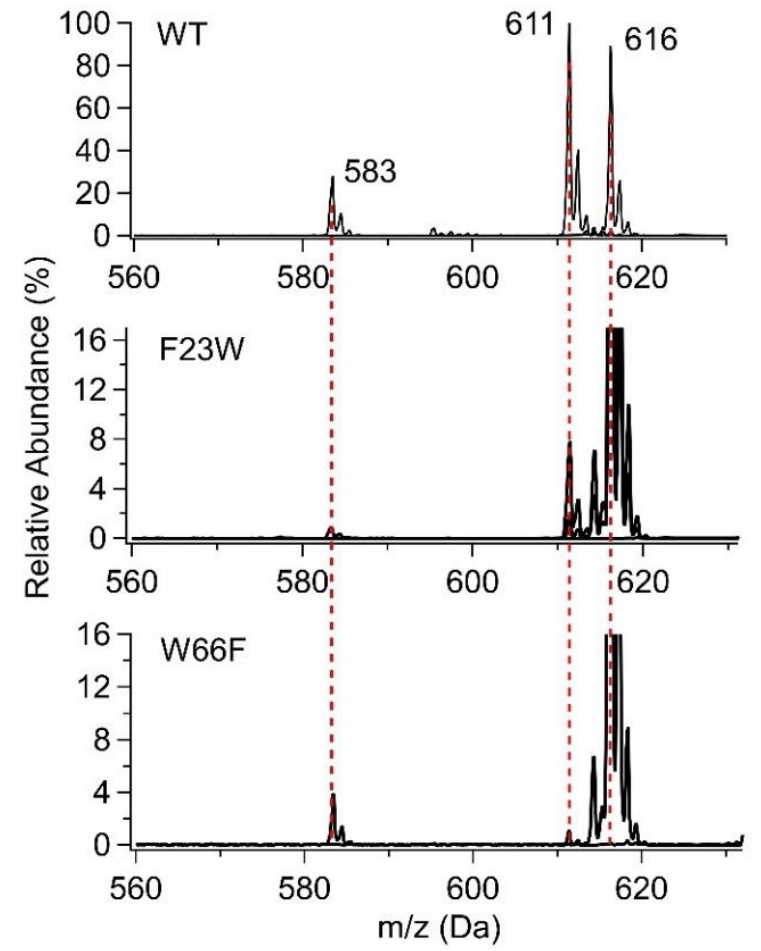

Figure 3. ESI-MS reveals that MhuD degrades heme to a mixture of mycobilin and biliverdin, and the ratio depends upon second-sphere interactions within the enzyme active site. WT MhuD degrades heme ( $\mathrm{m} / \mathrm{z} 616)$ to a major mycobilin product ( $\mathrm{m} / \mathrm{z}$ 611) and minor biliverdin product ( $\mathrm{m} / \mathrm{z} 583$, top). The major product of $\mathrm{F}_{23} \mathrm{~W}$ MhuD is also mycobilin, but enzymatic turnover is severely impaired (middle). In contrast, the major product of W66F MhuD is biliverdin (bottom). ESI-MS data were acquired after $50 \mu \mathrm{M}$ MhuD-heme was permitted to react with $5 \mathrm{mM}$ ascorbate in $50 \mathrm{mM} \mathrm{KP}$ i pH 6.0 for $30 \mathrm{~min}$ at $37^{\circ} \mathrm{C}$.

The major product of the ruffled conformation is mycobilin. Surprisingly, ESI-MS data for the reaction mixtures revealed that WT, $\mathrm{F}_{23} \mathrm{~W}$, and W66F MhuD degraded heme to a mixture of products. WT, F23W, and W66F MhuD-heme were allowed to react with a 10o-fold excess of ascorbate for $30 \mathrm{~min}$ at $37^{\circ} \mathrm{C}$, as described above for the $\mathrm{UV} / \mathrm{Vis}$ Abs-detected assays. In contrast to previous studies that have isolated MhuD degradation products prior to MS analysis, ${ }^{19,27}$ here the crude reaction mixture was analyzed by in proteo LC-MS. ${ }^{29}$ LC-MS of the WT and F $23 \mathrm{~W}$ MhuD reaction mixtures revealed a major product with $\mathrm{m} / \mathrm{z} 611$ and a minor product with $\mathrm{m} / \mathrm{z} 583$ (Figure 3). In contrast, the major product ion for the W66F MhuD-catalyzed reaction had a molecular weight of $583 \mathrm{Da}$ and the 611 Da species was the minor product. As a control, the same reaction was carried out in the absence of enzyme yielding small amount of the 583 Da product and no detectable production of the 611 Da product (Figure $\mathrm{S}_{5}$ ). Thus, MhuD degrades heme to two products, with molecular weights of 611 and $583 \mathrm{Da}$, and the W66F substitution changes the identity of the major product.

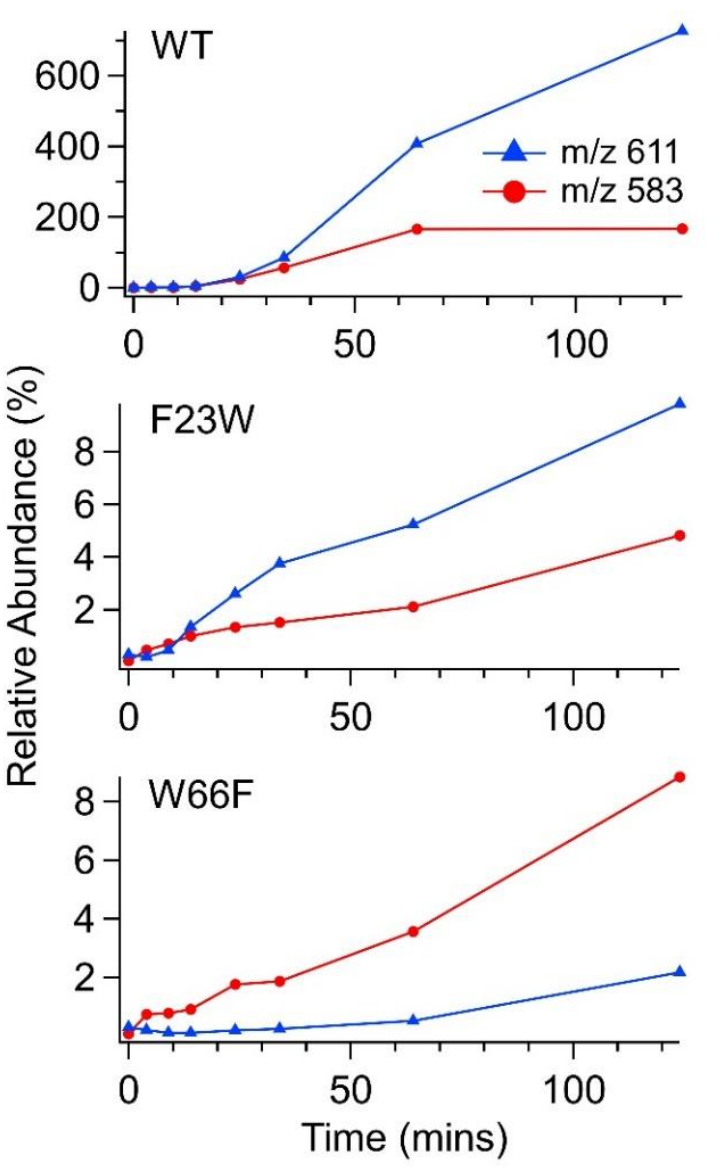

Figure 4. The production of mycobilin (m/z 611) and biliverdin ( $\mathrm{m} / \mathrm{z} 583)$ was monitored as a function of time for three variants of MhuD with altered second-sphere interactions. WT MhuD consistently produces mycobilin as a major product over the course of $2 \mathrm{~h}$ (top). F23W MhuD initially produces a larger fraction of biliverdin, but mycobilin production predominates after 20 min (middle). The major product of $\mathrm{W} 66 \mathrm{~F}$ MhuD is biliverdin (bottom). ESI-MS data were acquired for aliquots of a $37^{\circ} \mathrm{C}$ reaction between $50 \mu \mathrm{M}$ MhuD-heme and $5 \mathrm{mM}$ ascorbate in $50 \mathrm{mM} \mathrm{KP}$ i $\mathrm{pH}$ 6.o that were flash-frozen after o, 5, 10, 20, 30, 60, and 120 min. Mycobilin and biliverdin abundances are reported relative to the heme abundance at each time point.

Tandem LC-MS was employed to identify the 611 and 583 Da products of MhuD-catalyzed heme degradation as mycobilin and biliverdin, respectively. The tandem LC-MS fragmentation pattern for $\mathrm{m} / \mathrm{z} 616$ ion is very similar to that of authentic heme (Figure S6), identifying this species as unreacted substrate. Tandem LC-MS of the 611 Da product ion for WT enzyme yielded a major fragment ion at $\mathrm{m} / \mathrm{z}$ 476 along with several minor fragments (Figure $S_{7}$ ). The 476 Da fragment ion corresponds to fragmentation of mycobilin- $a$ between the $\delta$-meso carbon and pyrrole ring D, or fragmentation of mycobilin- $b$ between the $\beta$-meso carbon and pyrrole ring $C$. The minor fragment ions can also be assigned to reasonable fragmentations of mycobilin. Finally, tandem LC-MS was also performed for the $583 \mathrm{Da}$ product, yielding a single fragment ion at m/z 297 (Figure 
S8). This fragmentation was previously assigned to a biliverdin product of R26S MhuD-catalyzed heme degradation. ${ }^{27}$ Based upon these data, we can conclude that MhuD produces a mixture of mycobilin and biliverdin products.

Mycobilin and biliverdin production by WT, $\mathrm{F}_{23} \mathrm{~W}$, and W66F MhuD was monitored by ESI-MS to ascertain the influence of substrate conformation on product identity. The WT, F23W, and W66F MhuD reaction mixtures described above were flash-frozen at seven time points ranging from $\mathrm{o}$ to $120 \mathrm{~min}$, and the product distributions were analyzed by ESI-MS (Figure 4). Starting from the 20 min time point, the major product of WT MhuD is clearly mycobilin in accord with a previous study that relied upon product extraction. ${ }^{19}$ Prior to the 20 minute time point, mycobilin and biliverdin production by WT enzyme appears similar, but it is difficult to say whether this observation is due to different rates for the two heme degradation reactions or different ionization efficiencies for mycobilin and biliverdin. When population of the ruffled conformation was favored by the $\mathrm{F} 23 \mathrm{~W}$ substitution, mycobilin remained the major product but the rate of enzymatic turnover decreased dramatically. The $\mathrm{F}_{23} \mathrm{~W}$ MhuD mixture was also analyzed using Atmospheric pressure chemical ionization MS, which yielded evidence for generation of a 632 Da species, assigned to meso-hydroxyheme (Figure S9). Finally, when population of the planar conformation was favored by the W66F substitution, the major heme degradation product was biliverdin starting from the $5 \mathrm{~min}$ time point. Thus, the major heme degradation product for $\mathrm{WT}$ and $\mathrm{F}_{23} \mathrm{~W}$ MhuD is mycobilin, as previously reported for WT enzyme, ${ }^{19}$ but when the population of the planar substrate conformation is increased by the W66F substitution, biliverdin becomes the major enzyme product.

The major product of the planar conformation is $\alpha$ biliverdin. Closer analysis of the minor product for WT enzyme revealed four isomers that could be separated by LC. The LC gradient was optimized to separate four $\mathrm{m} / \mathrm{z}$ 583 species with retention times of $38.7,39.0,39.4$, and 40.2 min (Figure 5). The species with 38.7, 39.0, and $39.4 \mathrm{~min}$ retention times could be confidently assigned to $\alpha-, \delta$-, and $\beta$-biliverdin, respectively, based upon comparison of tandem LC-MS data to previously published data for $\mathrm{HOs}$ from Pseudomonas aeruginosa (Figure S10).32,33 The m/z 583 species with a 40.2 min retention time was also analyzed by tandem LC-MS yielding a major fragment ion at $\mathrm{m} / \mathrm{z} 555$ and a minor fragment at $\mathrm{m} / \mathrm{z} 297$, which is consistent with assignment of this species to $\gamma$-biliverdin. When the ruffled conformation was favored by introducing the F23W substitution, a mixture of $\alpha$-, $\delta$-, and $\beta$-biliverdin was observed. Although $\gamma$-biliverdin production by $\mathrm{F}_{23} \mathrm{~W}$ MhuD was minimal, it is important to note that the distal side of the $\gamma$-meso carbon is expected to be blocked by the Trp23 side-chain in the $\mathrm{F}_{23} \mathrm{~W}$ variant. ${ }^{10}$ Altogether, these data reveal that the biliverdin generated by the ruffled heme conformation is a mixture of regioisomers.

When the planar substrate conformation was stabilized by the W66F substitution, tandem LC-MS identified the major reaction product as $\alpha$-biliverdin. LC separation of the biliverdin isomers for W66F MhuD identified a major

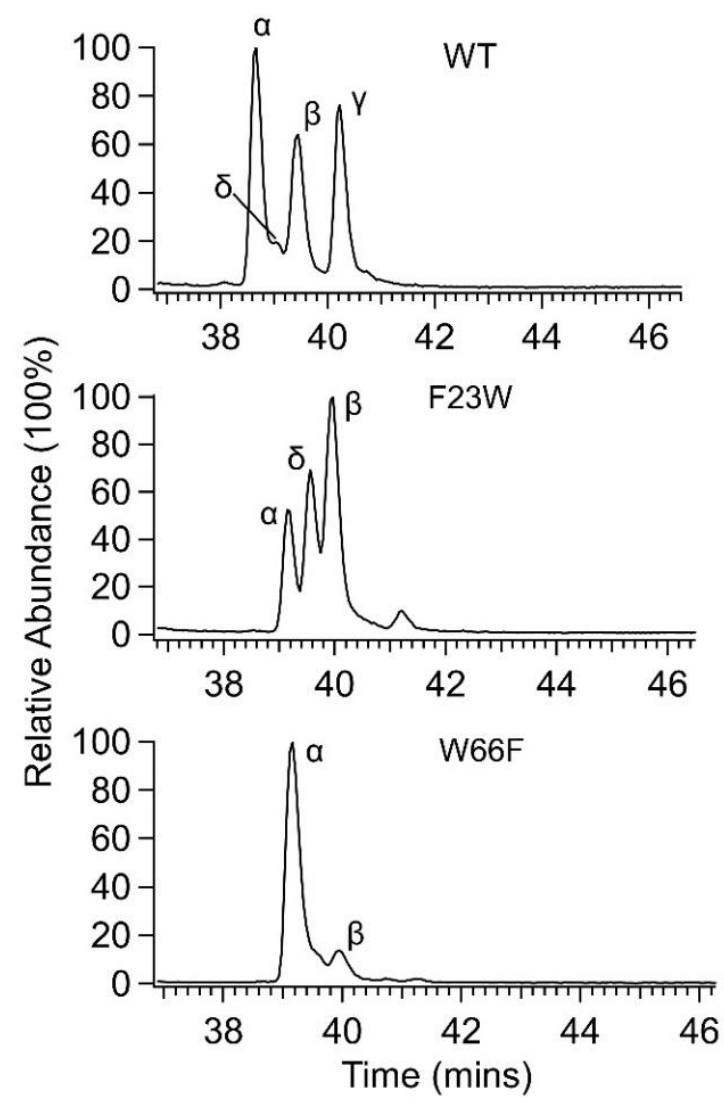

Figure 5. Tandem LC-MS reveals that second-sphere substitutions in the MhuD active site change the isomer(s) of biliverdin produced by the enzyme. WT MhuD produces a mixture of all four biliverdin isomers (top). F23W MhuD-catalyzed heme degradation also yields a mixture of biliverdin isomers, and the minimal production of $\gamma$-biliverdin may be due to steric protection of the $\gamma$-meso carbon of porphyrin by Trp23 (middle). The major product of W66F MhuD-catalyzed heme degradation is $\alpha$-biliverdin (bottom). Biliverdin isomers were resolved by reversed-phase liquid chromatography with a $\mathrm{C}_{18}$ column and a mobile phase linear gradient of acetonitrile in water.

product with a retention time of $39.1 \mathrm{~min}$ and a minor product with a retention time of 39.9 min (Figure 5). The assignments of these species to biliverdin isomers based upon retention time alone was ambiguous, so tandem LCMS was used to identify the two biliverdin isomers produced by W66F variant. These experiments identified the major product to be $\alpha$-biliverdin and the minor product to be $\beta$-biliverdin. It should be noted that $\alpha$-biliverdin is also the major heme degradation product for R26S MhuD. ${ }^{27}$ These observations suggest that $\alpha$-biliverdin production by R26S and W66F MhuD may be an enzymatic reaction, albeit one that proceeds at a slower rate than mycobilin production by WT enzyme. Since the W66F substitution is known to favor population of the planar substrate conformation, ${ }^{11}$ these data identify a correlation between population of the planar substrate conformation and $\alpha$-biliverdin production. 


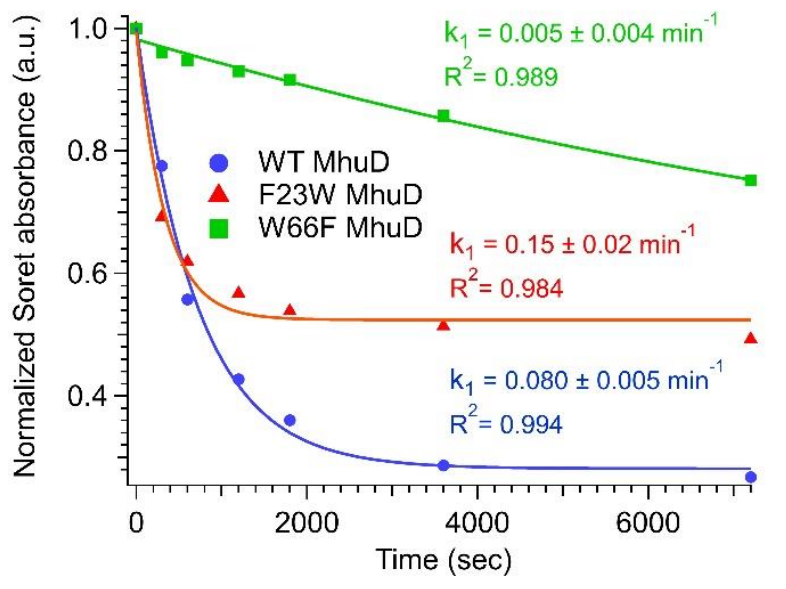

Figure 6. The pseudo-first order rate of heme monooxygenation by MhuD is correlated with population of the ruffled heme conformation. The MhuD variant housing the most ruffled heme, F23W MhuD, has the fastest heme monooxygenation rate. The MhuD variant housing the least ruffled heme, W66F MhuD, has the slowest heme monooxygenation rate. The three curves do not reach the same absorption intensity after $2 \mathrm{~h}$ due to spectral differences between the products of these three enzyme variants. The UV/Vis Abs data depicted in Figure 2 were fit to equation 1.

The ruffled conformation promotes heme monooxygenation. A kinetic model for heme monooxygenation was developed that accounts for the spectral differences between the WT, F23W, and W66F MhuD reaction products. Heme monooxygenation by MhuD is formally a thirdorder reaction dependent upon the concentrations of heme, molecular oxygen, and reductant. ${ }^{20}$ However, under the reaction conditions employed in this study, the reaction was pseudo-first order due to a molar excess of molecular oxygen and reductant. Previous studies have employed a pseudo-first order kinetic model to extract a rate constant for this rate-limiting step of heme degradation by non-canonical HOs. ${ }^{11,23}$ However, the observations of different enzyme products for different enzyme variants reported here identifies a critical flaw in the equation used to model the data since it assumes that the products have zero absorbance at the Soret band wavelength (Figure 2). Here, as detailed in the Supporting Information, we have derived equations to model pseudo-first order heme monooxygenation by WT, F23W, and W66F MhuD in a consistent manner. This will allow us to make a meaningful comparison of the heme monooxygenation rates for each enzyme variant.

Analyses of the UV/Vis Abs data within these frameworks revealed that increased population of the ruffled substrate conformation resulted in a faster heme monooxygenation rate. When the UV/Vis Abs data was analyzed for WT enzyme using equation (1), the analysis yielded a rate of $0.080 \pm 0.005 \mathrm{~min}^{-1}$ for heme monooxygenation (Figure 6). The same analysis was applied to the UV/Vis Abs data for $\mathrm{F}_{23} \mathrm{~W}$ MhuD, which favors the ruffled heme conformation, and a pseudo-first order rate constant of $0.15 \pm$ $0.02 \mathrm{~min}^{-1}$ was obtained. Finally, the analysis was repeated
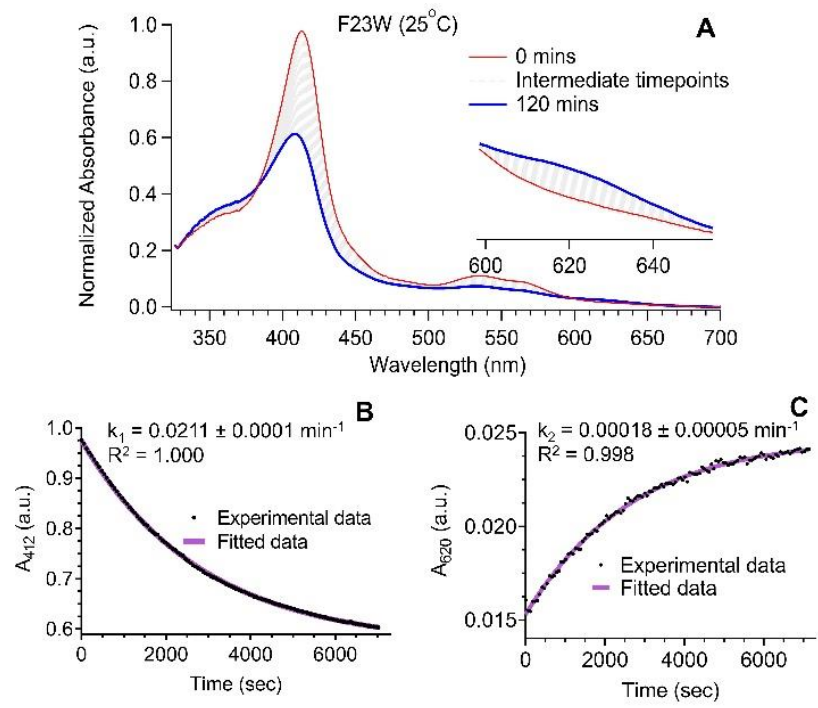

Figure 7. F23W MhuD-catalyzed heme degradation was slowed by decreasing the temperature to $25{ }^{\circ} \mathrm{C}$ yielding improved data resolution for build-up of the meso-hydroxyheme intermediate. Spectral data were acquired every 1 min for 120 min. The o min trace is solid red, the 120 min trace solid blue, and the 1-119 min traces are dashed gray lines (panel A). Kinetic analyses of the time-courses for UV/Vis absorption at 412 $\mathrm{nm}$ (panel B) and $620 \mathrm{~nm}$ (panel C) revealed that the $\mathrm{F}_{23} \mathrm{~W}$ substitution changes the rate-limiting step of heme degradation by MhuD. The $412 \mathrm{~nm}$ and $620 \mathrm{~nm}$ data were fit to equations 1 and 2, respectively.

for the W66F variant, which favors the planar heme conformation, yielding a pseudo-first order rate constant of $0.005 \pm 0.004 \mathrm{~min}^{-1}$. These data were also analyzed using equation (2), which explicitly considers the contribution of meso-hydroxyheme to the UV/Vis Abs spectrum observed for F23W MhuD (Figure 2). The heme monooxygenation rates extracted by the two kinetic models for WT and W66F MhuD were within error of one another (Figure S11). However, for $\mathrm{F}_{23} \mathrm{~W}$ MhuD, the monooxygenation rate extracted using equation (2) was approximately double that obtained with equation (1) and the $\mathrm{R}^{2}$ value of the fit was significantly improved. Thus, inclusion of a term to explicitly model the absorbance of meso-hydroxyheme is only necessary for the F23W variant. Overall, these data identify a clear correlation between increased population of the ruffled heme conformation and an increased heme monooxygenation rate.

The planar conformation promotes substrate dioxygenation. A kinetic model was developed to describe the time course of the meso-hydroxyheme intermediate created by heme monooxygenation and degraded by dioxygenation to mycobilin. In the presence of molar excesses of molecular oxygen and reductant, heme oxygenation to mycobilin via meso-hydroxyheme can be described by two consecutive pseudo-first order reactions. ${ }^{19}$ The initial pseudo-first order reaction forms meso-hydroxyheme from monooxygenation of heme with a rate constant $k_{1}{ }^{20}$ This is the same heme monooxygenation reaction that was 
analyzed above, and $k_{1}$ was constrained to the value determined by fitting the time course of the Soret band to equation (1). The second pseudo-first order reaction is dioxygenation of meso-hydroxyheme to mycobilin with a rate constant of $k_{2}$. As detailed in the Supporting Information, a kinetic model was derived for the formation and degradation of meso-hydroxyheme where the only unknown values were $k_{2}$ and the molar extinction coefficient of MhuDbound meso-hydroxyheme. By analyzing the time-course of the $620 \mathrm{~nm}$ band observed for F23W MhuD, we will be able to estimate $k_{2}$ for this enzyme variant.

Analyses of the UV/Vis Abs data within the framework of this model revealed that increased population of the planar substrate conformation yielded a faster dioxygenation rate. In order to improve the time resolution of the data, the UV/Vis Abs assay for $\mathrm{F}_{23} \mathrm{~W}$ MhuD was repeated at room temperature under otherwise similar conditions (Figure 7). Analysis of the Soret band decrease for $\mathrm{F}_{23} \mathrm{~W}$ MhuD at room temperature yielded a heme monooxygenation rate $\left(k_{1}\right)$ of $0.0211 \pm 0.0001 \mathrm{~min}^{-1}$, which is expectedly slower than the reaction carried out at $37^{\circ} \mathrm{C}$. Notably, the $\mathrm{F}_{23} \mathrm{~W}$ MhuD-catalyzed reaction is slow enough at $25^{\circ} \mathrm{C}$ to effectively model the spectral changes at $412 \mathrm{~nm}$ using equation (1). Next, the formation and degradation of mesohydroxyheme band was satisfactorily modelled by the sequential first-order kinetic model where the rate of mesohydroxyheme formation was set to be equal to the rate of heme monooxygenation. By fitting the time course of the $620 \mathrm{~nm}$ Abs band to this model, we extracted a $k_{2}$ of $0.00018 \pm 0.00005 \mathrm{~min}^{-1}$ for $\mathrm{F}_{23} \mathrm{~W}$ MhuD-catalyzed mesohydroxyheme degradation. Since meso-hydroxyheme formation by WT and W66F MhuD was not observed under similar conditions, it can be concluded that the F23W substitution has changed the rate-limiting step of the reaction. The F $23 \mathrm{~W}$ substitution decreases population of the planar substrate conformation, ${ }^{12}$ so there is a correlation between population of the planar substrate conformation and efficient meso-hydroxyheme dioxygenation.

\section{DISCUSSION}

The identity of the MhuD product depends upon the substrate conformation. The data presented in this article for $\mathrm{F}_{23} \mathrm{~W}$ MhuD, a variant that stabilizes the ruffled conformation, clearly demonstrates that MhuD converts ruffled heme to mycobilin. The kinetic analysis presented above reveals that population of the ruffled conformation favors rapid heme monooxygenation since $\mathrm{F}_{23} \mathrm{~W}$ MhuD has the largest $k_{1}$ (Figure 6). Based upon the observation that mycobilin is the major product of $\mathrm{F} 23 \mathrm{~W}$ MhuD (Figure 4 ), and the report that only the $\beta$ and $\delta$ isomers of mesohydroxyheme are converted to mycobilin by $\mathrm{MhuD},{ }^{20}$ MhuD-catalyzed monooxygenation of ruffled heme is regiospecific for the $\beta$ - and $\delta$-meso carbons of the porphyrin ring. However, dioxygenation by $\mathrm{F}_{23} \mathrm{~W}$ MhuD is extremely slow (Figure 7), which means that MhuD cannot rapidly dioxygenate ruffled meso-hydroxyheme to mycobilin. Most likely, this is due to a ruffling-induced electronic structure change for meso-hydroxyheme, ${ }^{19}$ whose anion

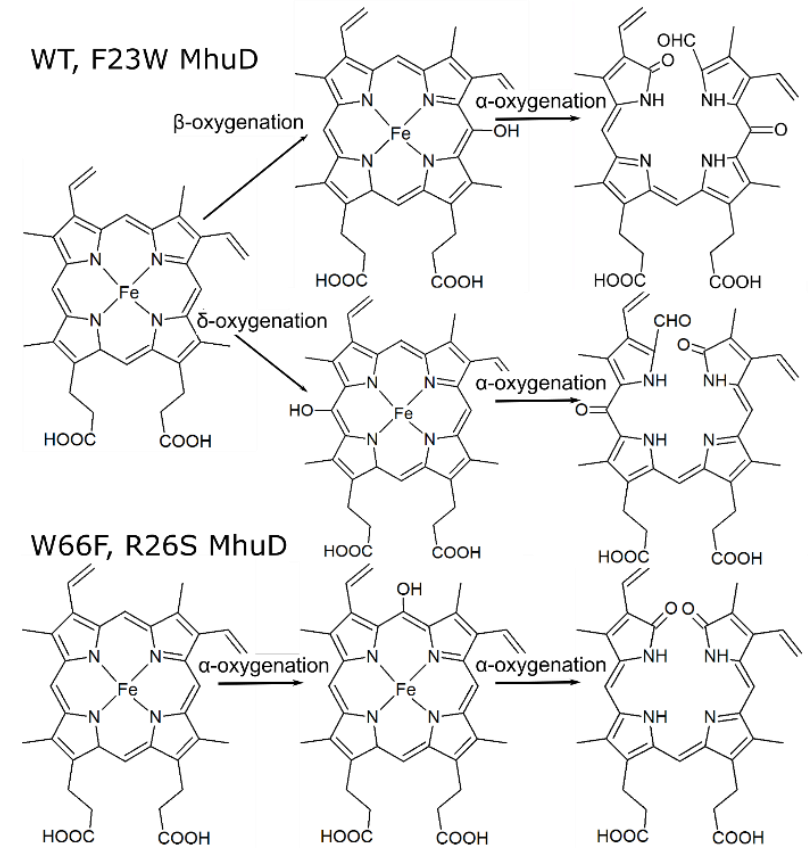

Figure 8. The regioselectivity of MhuD-catalyzed heme monooxygenation depends upon second-sphere interactions in the enzyme active site, but all characterized MhuD variants catalyze $\alpha$-oxygenation of meso-hydroxyheme. Based upon the data presented here, WT and F23W MhuD catalyze monooxygenation of the $\beta$ - and $\delta$-meso carbons of heme en route to mycobilins. W66F MhuD catalyzes regiospecific oxygenation of the $\alpha$-meso carbon, which was previously observed for R26S MhuD. ${ }^{20}$

can best be described as: ferric porphyrin anion ketone, ferric porphyrin enolate, or ferrous porphyrin hydroxyl radical depending upon the relative energies of the ironand porphyrin-based orbitals. ${ }^{34,35}$ Finally, the minor products of MhuD-catalyzed ruffled heme degradation, a mixture of biliverdin isomers (Figure 5), can be attributed to coupled oxidation of heme. ${ }^{36}$ It is important to once again note that the physiological redox partner for MhuD has not yet been identified, and these side products could be artifacts arising from an overpotential for ascorbate or lack of a structural change induced by the protein-protein complex. Thus, the ruffled substrate conformation supports rapid heme monooxygenation to meso-hydroxyheme by MhuD, but this conformation alone is not sufficient for rapid oxygenation of heme to mycobilin.

On the other hand, the data presented here for the W66F variant, which favors the planar substrate conformation, reveals that MhuD degrades planar heme to $\alpha$-biliverdin. The major product of W66F MhuD-catalyzed heme degradation is $\alpha$-biliverdin (Figure 5), which is only oxygenated at the $\alpha$-meso carbon position, so W66F MhuD-catalyzed heme monooxygenation must proceed through an $\alpha$-mesohydroxyheme intermediate (Figure 8). The rate of $\alpha$-meso carbon hydroxylation of planar heme by MhuD is at least 30 times slower than the rate of $\beta / \delta$-meso carbon hydroxylation by ruffled heme (Figure 6), which explains why mycobilin is the major product of WT enzyme where both conformations are accessible. ${ }^{10,12}$ The meso-hydroxyheme 
intermediate was not observed for UV/Vis Abs-monitored heme degradation by WT or W66F MhuD (Figure 2), indicating that this is not the rate-limiting step for degradation of planar heme by MhuD and $k_{2}>0.080 \pm 0.005 \mathrm{~min}^{-1}$. For comparison, the rate of meso-hydroxyheme oxygenation by rat $\mathrm{HO}$ is $4,200 \mathrm{~min}^{-1}$ under similar conditions. ${ }^{37} \alpha$-meso carbon oxygenation of planar meso-hydroxyheme by MhuD is rapid. Finally, the observation of mycobilin as a minor product for W66F MhuD-catalyzed heme degradation can be explained by limited access to the ruffled substrate conformation in this variant. The heme substrate of W66F MhuD exists in a dynamic equilibrium between planar and ruffled heme that favors planar heme, but does not fully exclude ruffled heme. ${ }^{11}$ Despite the fact that MhuD converts heme to $\alpha$-biliverdin, this substrate conformation does facilitate the second oxygenation reaction catalyzed by the enzyme, namely, $\alpha$-meso carbon oxygenation of meso-hydroxyheme.

A proposed mechanism for heme oxygenation by MhuD. The data presented in this article allows us to propose a more detailed mechanism for MhuD-catalyzed heme monooxygenation than was previously available. Based upon a previous study where researchers fed synthetic meso-hydroxyheme to MhuD, the mechanistic proposal prior to this publication was that MhuD catalyzes regioselective monooxygenation of heme to $\beta$ - and $\delta$-mesohydroxyheme. ${ }^{20}$ A recent hybrid quantum mechanics / molecular mechanics (QM/MM) study attributed the regioselectivity of MhuD-catalyzed heme monooxygenation to a putative hydrogen bond between Asn 7 and the terminal oxygen of the putative ferric-hydroperoxoheme intermediate. $3^{8}$ The UV/Vis Abs data presented here identifies another structural consideration for regioselective heme monooxygenation by MhuD. To be specific, the kinetic analysis of the heme monooxygenation reaction reveals that heme must adopt a ruffled conformation prior to monooxygenation (Figure 9). Interestingly, the trend where $k_{1}$ increases with increasing heme ruffling cannot be explained by steric considerations because the ruffling deformation induced by the MhuD active site pushes the $\beta$ and $\delta$-meso carbons away from a distal hydroperoxo ligand and towards the proximal His (PDB ID $4 \mathrm{NL} 5) .{ }^{10}$ Instead, the monooxygenation rate enhancement is likely derived from an electronic structure change similar to that previously reported for IsdG. 39 These data elucidate a mechanism where a dynamic equilibrium between planar and ruffled heme exists within the MhuD active site, ${ }^{12}$ but heme monooxygenation only proceeds for the ruffled conformation.

The data presented here also demands a revision of the proposed mechanism for meso-hydroxyheme dioxygenation by MhuD. The same synthetic meso-hydroxyheme study noted above also revealed that MhuD catalyzes dioxygenation of the $\alpha$-meso carbon of $\beta$ - and $\delta$-meso-hydroxyheme to mycobilin- $a$ and $-b,{ }^{20}$ and attributed the novel reactivity from canonical HOs to a ruffling-induced electronic structure change. $4^{40,41}$ The QM/MM study noted above developed a model for this proposed reaction by pre
Planar

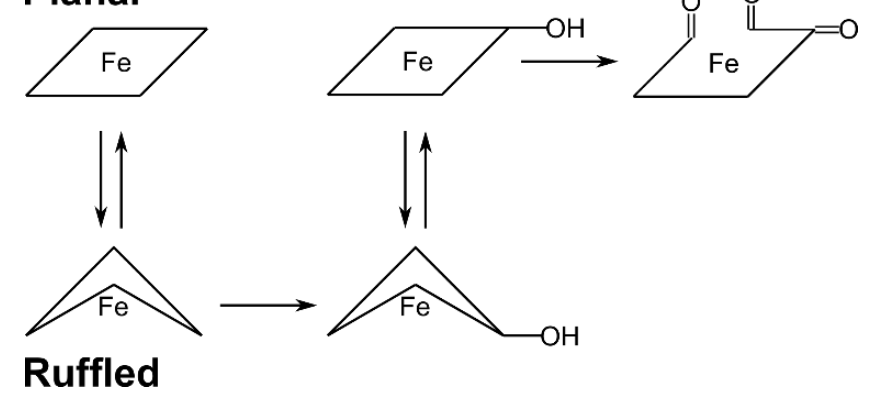

Figure 9. Proposed mechanism for MhuD-catalyzed heme oxygenation. Following heme substrate binding, an equilibrium is established between planar and ruffled heme. MhuD monooxygenates ruffled heme, and an equilibrium is established between planar and ruffled meso-hydroxyheme. Finally, MhuD catalyzes dioxygenation of planar meso-hydroxyheme.

dicting the energy changes associated with adding molecular oxygen to the $\alpha$-meso carbon of ruffled $\delta$-meso-hydroxyheme. ${ }^{38}$ In agreement with this model, the MS data presented in this article strongly suggests that all MhuDcatalyzed oxygenations of meso-hydroxyheme occur at the $\alpha$-meso carbon position (Figure 8 ). However, the UV/Vis Abs and MS data presented in this article indicate that meso-hydroxyheme must adopt a planar conformation prior to further oxygenation (Figure 9). A likely source of the discrepancy between the experimental data presented here and the computational data in the literature is that the setup of the QM/MM calculations froze the protein in the conformation observed for MhuD-heme-CN (PDB ID $\left.{ }_{4} \mathrm{NL}_{5}\right),{ }^{10}$ but at least three other protein conformations exist.13,14 The novel reactivity of the meso-hydroxyheme form of MhuD could still be due to an electronic structure change, but this electronic structure change is not derived from substrate ruffling. Alternatively, the MhuD active site could catalyze meso-hydroxyheme dioxygenation in a manner similar to cofactor-independent oxygenases, ${ }^{42}$ with Streptomyces coelicolor ActVA-Orf6 being a particularly relevant precedent due to the structural homology of MhuD and ActVA-Orf6. ${ }^{33,43,44}$ Thus, although this study provides significant new insight into the mechanism of meso-hydroxyheme dioxygenation by MhuD, several important open questions remain.

MhuD relies upon a dynamic, out-of-plane, substrate distortion. Perhaps the single most important contribution of the data presented in this article to our understanding of the MhuD mechanism is that enzymatic turnover requires a dynamic substrate. The heme monooxygenation reaction catalyzed by MhuD requires access to the ruffled substrate conformation. This conformation is derived from a pseudo Jahn-Teller distortion that mixes ${ }^{2} A_{2 u}$ character into the ${ }^{2} B_{2 g}$ ground state yielding partial radical character on the porphyrin meso carbons and promoting their combination with a hydroxyl radical generated in situ (Figure 10).12,15,16 However, this correlation between heme out-of-plane ruffling and electronic structure is specific for low-spin ferric heme. Meso-hydroxyheme can be described as a ferrous oxyheme with a porphyrin-based radical.34,35 


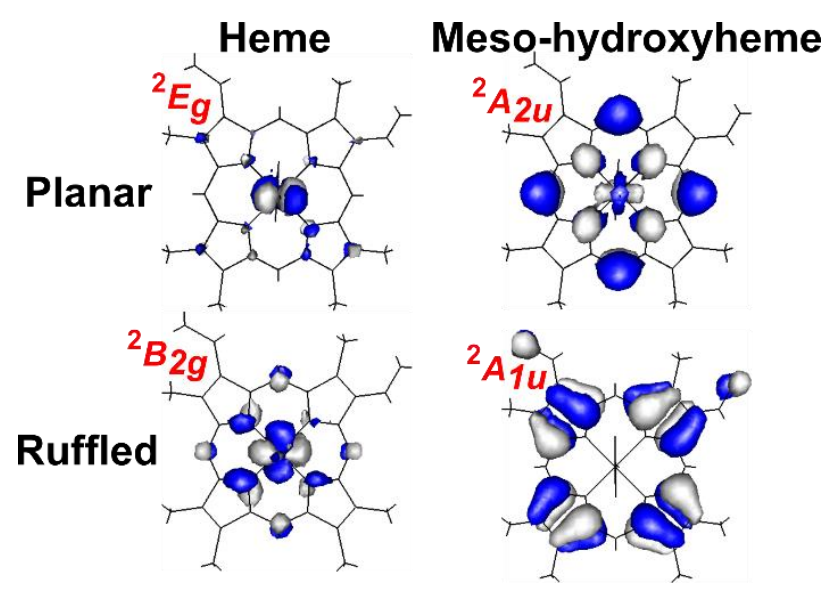

Figure 10. Expected singly-occupied molecular orbitals for planar and ruffled heme and meso-hydroxyheme, and the corresponding electronic states. To place spin density on the heme meso carbons, heme must adopt a ruffled conformation. ${ }^{11}$ Conversely, meso-hydroxyheme must adopt a planar conformation to place spin density on the meso carbons. Note, the ${ }^{2} E_{g}$ state is doubly-degenerate and only one of the $e_{g}$ orbitals is shown.

The pseudo Jahn-Teller vibronic mixing described above lowers the energy of the ${ }^{2} B_{2 g}$ state at the expense of raising the energy of the ${ }^{2} A_{2 u}$ state. This means that ruffled mesohydroxyheme is likely to have ${ }^{2} A_{1 u}$ character with the radical localized to the porphyrin pyrrole carbons, the planar meso-hydroxyheme is likely to have ${ }^{2} A_{2 u}$ character with the radical localized to the porphyrin meso carbons, and mesohydroxyheme oxygenation is most favorable for a planar substrate. ${ }^{45,46}$ In essence, the bifunctionality of MhuD requires access to two substrate conformations because these conformations differentially tune the electronic structures of the heme substrate and the meso-hydroxyheme intermediate.

The data presented in this article have revealed that heme dynamics are an essential consideration for MhuDcatalyzed heme degradation, and further consideration for the role of heme dynamics in all heme-dependent proteins is warranted. Ever since the types of heme out-of-plane distortion were categorized over 20 years ago, ${ }^{8}$ correlations between out-of-plane heme distortion and protein function have been regularly identified.9,47 However, what has not been as generally appreciated is that the out-of-plane vibrational modes are on the order of thermal energy at 37 ${ }^{\circ} \mathrm{C}(220 \mathrm{~cm}-1) \cdot{ }^{17,18}$ For MhuD, this means that the heme substrate of WT enzyme is regularly interconverting between the planar and ruffled substrate conformations. ${ }^{12}$ In general, the energy required to trigger an out-of-plane heme distortion means that we need to envision protein-bound heme as continually undergoing dynamic out-of-plane distortion from the equilibrium geometry captured by X-ray crystallography. As dramatically exemplified by heme oxygenases, ${ }^{10,22}$ these structural dynamics are tied to electron dynamics that significantly influence heme reactivity. Heme has a dense cluster of iron- and porphyrin-based orbitals near the HOMO/LUMO gap, ${ }^{46}$ and subtle structural changes can alter the orbital occupations resulting in dramatic changes to heme reactivity. Thus, heme cannot be considered a classic organic substrate where static structure determines function, and the influence of heme dynamics on heme electronic structure represents an exciting open challenge for physical bioinorganic chemistry.

\section{CONCLUSIONS}

In conclusion, this article presents new data which reveals that a dynamic heme substrate is critical for MhuDcatalyzed heme degradation. UV/Vis Abs and MS data for MhuD variants that favor either a ruffled or planar substrate conformation have shown that the MhuD product depends upon the substrate conformation. Single-turnover kinetic analyses of the UV/Vis Abs data have demonstrated that the rates of heme monooxygenation and meso-hydroxyheme dioxygenation also depend upon the substrate conformation. These observations have enabled us to prepare a refined enzymatic mechanism that is consistent with both previously published data and the new data presented here. This mechanism relies upon a dynamic heme substrate and raises the question of whether heme dynamics should be more carefully considered as a significant contributor to heme function.

\section{ASSOCIATED CONTENT}

Supporting Information. FPLC traces, SDS-PAGE gels, MS data, and complete derivations of equations 1 and 2. This material is available free of charge via the Internet at http://pubs.acs.org.

Accession Codes. MhuD, $\mathrm{P}_{9} \mathrm{WKH}_{3}$.

\section{AUTHOR INFORMATION}

\section{Corresponding Author}

*E-mail for M.D.L.: matthew.liptak@uvm.edu

ORCID

Matthew D. Liptak: oooo-ooo2-4951-6636

Funding

M.D.L. thanks the National Institutes of Health (Ro1GM114277) for financial support.

Notes

The authors declare no competing financial interest.

\section{ACKNOWLEDGMENT}

The authors acknowledge Celia Goulding (University of California-Irvine) for helpful discussions and a critical reading of the manuscript.

\section{REFERENCES}

(1) Jarrett, J. T.; Huang, S.; Matthews, R. G. Methionine synthase exists in two distinct conformations that differ in reactivity toward methyltetrahydrofolate, adenosylmethionine, and flavodoxin, Biochemistry 1998, 37, 5372-5382.

(2) Bandarian, V.; Ludwig, M. L.; Matthews, R. G. Factors modulating conformational equilibria in large modular proteins: a case study with cobalamin-dependent methionine synthase, Proc. Natl. Acad. Sci. 2003, 100, 8156-8163. 
(3) Evans, J. C.; Huddler, D. P.; Hilgers, M. T.; Romanchuk, G.; Matthews, R. G.; Ludwig, M. L. Structures of the N-terminal modules imply large domain motions during cataysis by methionine synthase, Proc. Natl. Acad. Sci. 2004, 101, 3729-3736.

(4) Monod, J.; Wyman, J.; Changeux, J.-P. On the nature of allosteric transitions: a plausible model, J. Mol. Biol. 1965, 12, 88-118.

(5) Perutz, M. F. Stereochemistry of Cooperative Effects in Haemoglobin, Nature 1970, 228, 726-734.

(6) Yuan, Y.; Tam, M. F.; Simplaceanu, V.; Ho, C. New look at hemoglobin allostery, Chem. Rev. 2015, 115, 1702-1724.

(7) Jentzen, W.; Simpson, M. C.; Hobbs, J. D.; Song, X.; Ema, T.; Nelson, N. Y.; Medforth, C. J.; Smith, K. M.; Veyrat, M.; Mazzanti, M.; Ramasseul, R.; Marchon, J.-C.; Takeuchi, T.; Goddard, W. A.; Shelnutt, J. A. Ruffling in a series of nickel(II) meso-tetrasubstituted porphyrins as a model for the conserved ruffling of the heme of cytochromes c, J. Am. Chem. Soc. 1995, 117, 11085-11097.

(8) Jentzen, W.; Song, X.-Z.; Shelnutt, J. A. Structural characterization of synthetic and protein-bound porphyrins in terms of the lowest-frequency normal coordinates of the macrocycle, $J$. Phys. Chem. B 1997, 101, 1684-1699.

(9) Shelnutt, J. A.; Song, X.-Z.; Ma, J.-G.; Jia, S.-L.; Jentzen, W.; Medforth, C. J. Nonplanar Porphyrins and their Significance in Proteins, Chem. Soc. Rev. 1998, 27, 31-41.

(10) Graves, A. B.; Morse, R. P.; Chao, A.; Iniguez, A.; Goulding, C. W.; Liptak, M. D. Crystallographic and spectroscopic insights into heme degradation by Mycobacterium tuberculosis MhuD, Inorg. Chem. 2014, 53, 5931-5940.

(11) Graves, A. B.; Graves, M. T.; Liptak, M. D. Measurement of heme ruffling changes in MhuD using UV/Vis spectroscopy, $J$. Phys. Chem. B 2016, 120, 3844-3853.

(12) Graves, A. B.; Horak, E. H.; Liptak, M. D. Dynamic ruffling distortion of the heme substrate in non-canonical heme oxygenase enzymes, Dalton Trans. 2016, 10058-10067.

(13) Chim, N.; Iniguez, A.; Nguyen, T. Q.; Goulding, C. W. Unusual diheme conformation of the heme-degrading protein from Mycobacteriuim tuberculosis, J. Mol. Biol. 2010, 395, 595-6o8.

(14) Chao, A.; Burley, K. H.; Sieminski, P. J.; de Miranda, R.; Chen, X.; Mobley, D. L.; Goulding, C. W. Structure of a Mycobacterium tuberculosis heme-degrading protein, $\mathrm{MhuD}$, variant in complex with its product, Biochemistry 2019, 58, 4610-4620.

(15) Garcia-Fernandez, P.; Bersuker, I. B.; Boggs, J. E. Orbital disproportionation and spin crossover as a pseudo Jahn-Teller effect, J. Chem. Phys. 2006, 125, 104102.

(16) Bersuker, I. B. Pseduo-Jahn-Teller Effect-a two-state paradigm in formation, deformation, and transformation of molecular systems and solids, Chem. Rev. 2013, 113, 1351-1390.

(17) Kubo, M.; Gruia, F.; Benabbas, A.; Barabanschikov, A.; Montfort, W. R.; Maes, E. M.; P.M., C. Low-frequency mode activity of heme: femtosecond coherence spectroscopy of iron porphine halides and nitrophorin, J. Am. Chem. Soc. 2008, 130, 980o9811.

(18) Karunakaran, V.; Sun, Y.; Benabbas, A.; Champion, P. M. Investigations of the low frequency modes of ferric cytochrome $c$ using vibrational coherence spectroscopy, J. Phys. Chem. B 2014, 118.

(19) Nambu, S. M., T.; Goulding, C.W.; Takahashi, S.; IkedaSaito, M. A new way to degrade heme: the Mycobacterium tuberculosis enzyme MhuD catalyzes heme degradation without generating CO, J. Biol. Chem. 2013, 288, 10101-10109.

(20) Matsui, T.; Nambu, S.; Goulding, C. W.; Takahashi, S.; Fujii, H.; Ikeda-Saito, M. Unique coupling of mono- and dioxygenase chemistries in a single active site promotes heme degradation, Proc. Natl. Acad. Sci. 2016, 113, 3779-3784.

(21) Matsui, T.; Unno, M.; Ikeda-Saito, M. Heme oxygenase reveals its strategy for catalyzing three successive oxygenation reactions, Acc. Chem. Res. 2010, 43, 240-247.
(22) Takayama, S. J.; Ukpabi, G.; Murphy, M. E.; Mauk, A. G. Electronic properties of the highly ruffled heme bound to the heme degrading enzyme IsdI, Proc. Natl. Acad. Sci. 2011, 108, 13071-13076.

(23) Ukpabi, G.; Takayama, S. J.; Mauk, A. G.; Murphy, M. E. Inactivation of the heme degrading enzyme IsdI by an active site substitution that diminishes heme ruffling, J. Biol. Chem. 2012, 287, 34179-34188.

(24) Angeli, C.; Cimiraglia, R.; Evangelisti, S.; Leininger, T.; Malrieu, J.-P. Introdution of $n$-electron valence states for multireference perturbation theory, J. Chem. Phys. 2001, 114, 1025210264.

(25) Angeli, C.; Cimiraglia, R.; Malrieu, J.-P. N-electron valence state perturbation theory: a fast implementation of the strongly contracted variant, Chem. Phys. Lett. 2001, 350, 297-305.

(26) Angeli, C.; Cimiraglia, R.; Malrieu, J.-P. N-electron valence state perturbation theory: a spinless formulation and an efficient implementation of the strongly contracted and of the partially contracted variants, J. Chem. Phys. 2002, 117, 9138-9153.

(27) Chao, A.; Goulding, C. W. A single mutation in the Mycobacterium tuberculosis heme-degrading protein, MhuD, results in different products, Biochemistry 2019, 58, 489-492.

(28) Thakuri, B.; Graves, A. B.; Chao, A.; Johansen, S. L.; Goulding, C. W.; Liptak, M. D. The affinity of MhuD for heme is consistent with a heme degrading function in vivo, Metallomics 2018, $10,1560-1563$.

(29) Bothner, B.; Chavez, R.; Wei, J.; Strupp, C.; Phung, Q.; Schneemann, A.; Siuzdak, G. Monitoring enzyme catalysis with mass spectrometery, J. Biol. Chem. 20oo, 275, 13455-13459.

(30) Berry, E. A.; Trumpower, B. L. Simultaneous Determination of Hemes $a, b$, and $c$ from Pyridine Hemochrome Spectra, Anal. Biochem. 1987, 161, 1-15.

(31) Wilks, A.; Ortiz de Montellano, P. R. Rat liver heme oxygenase: high level expression of a truncated soluble form and nature of the meso-hydroxylating species, J. Biol. Chem. 1993, 268, 2235722362.

(32) Barker, K. D.; Barkovits, K.; Wilks, A. Metabolic flux of extracellular heme uptake in Pseudomonas aeruginosa is driven by the iron-regulated heme oxygenase (HemO), J. Biol. Chem. 2o12, 287, 18342-18350.

(33) O'Neill, M. J.; Wilks, A. The $P$. aeruginosa heme binding protein PhuS is a heme oxygenase titratable regulator of heme uptake, ACS Chem. Biol. 2013, 8, 1794-1802.

(34) Matera, K. M.; Takahashi, S.; Fujii, H.; Zhou, H.; Ishikawa, K.; Yoshimura, T.; Rousseau, D. L.; Yoshida, T.; Ikeda-Saito, M. Oxygen and one reducing equivalent are both required for the conversion of $\alpha$-hydroxyhemin to verdoheme in heme oxygenase, J. Biol. Chem. 1996, 271, 6618-6624.

(35) Liu, Y.; Moenne-Loccoz, P.; Loehr, T. M.; Ortiz de Montellano, P. R. Heme oxygenase-1, intermediates in verdoheme formation and the requirement for reduction equivalents, J. Biol. Chem. 1997, 272, 6909-6917.

(36) Legge, J. W.; Lemberg, R. Coupled oxidation of ascorbic acid and haemoglobin: the 'labile iron' in blood and its increase in choleglobin formation, Biochem. J. 1941, 35, 353-362.

(37) Sakamoto, H.; Takahashi, S.; Higashimoto, Y.; Harada, S.; Palmer, G.; Noguchi, M. A kinetic study of the mechanism of conversion of $\alpha$-hydroxyheme to verdoheme while bound to heme oxygenase, Biochem. Biophys. Res. Commun. 2005, 338, 578-583.

(38) Yuan, C.; Zhang, Y.; Tan, H.; Li, X.; Chen, G.; Jia, Z. ONIOM investigations of the heme degradation mechanism by MhuD: the critical function of heme ruffling, Phys. Chem. Chem. Phys. 2020, 22, 8817-8826.

(39) Conger, M. A.; Cornetta, A. R.; Liptak, M. D. Spectroscopic evidence for electronic control of heme hydroxylation by IsdG, Inorg. Chem. 2019, 58, 15455-15465. 
(40) Morishima, I.; Fujii, H.; Shiro, Y.; Sano, S. Studies on the iron(II) meso-oxyporphyrin $\pi$-neutral radical as a reaction intermediate in heme catabolism, Inorg. Chem. 1995, 34, 1528-1535.

(41) Zhang, X.; Fujii, H.; Mansfield Matera, K.; Migita, C. T.; Sun, D.; Sato, M.; Ikeda-Saito, M.; Yoshida, T. Stereoselectivity of each of the three steps of the heme oxygenase reaction: hemin to meso-hydroxyhemin, meso-hydroxyhemin to verdoheme, and verdoheme to biliverdin, Biochemistry 2003, 42, 7418-7426.

(42) Fetzner, S.; Steiner, R. A. Cofactor-independent oxidases and oxygenases, Appl. Microbiol. Biotechnol. 2010, 86, 791-804.

(43) Sciara, G.; Kendrew, S. G.; Miele, A. E.; Marsh, N. G.; Federici, L.; Malatesta, F.; Schimperna, G.; Savino, C.; Vallone, B. The structure of ActVA-Orf6, a novel type of monooxygenase involved in actinorhodin biosynthesis, EMBO J. 2003, 22, 205-215.
(44) Skaar, E. P.; Gaspar, A. H.; Schneewind, O. IsdG and IsdI, heme-degrading enzymes in the cytoplasm of Staphylococcus aureus, J. Biol. Chem. 2004, 279, 436-443.

(45) Gouterman, M. Study of the effects of substitution on the absorption spectra of porphin, J. Chem. Phys. 1959, 30, 1139-1161.

(46) Walker, F. A. Magnetic spectroscopic (EPR, ESEEM, Mössbauer, MCD and NMR) studies of low-spin ferriheme centers and their corresponding heme proteins, Coord. Chem. Rev. 1999, 185186, 471-534.

(47) Senge, M. O.; MacGowan, S. A.; O'Brien, J. M. Conformational control of cofactors in nature - the influence of protein-induced macrocycle distortion on the biological function of tetrapyrroles, Chem. Commun. 2015, 51, 17031-17063. 
For Table of Contents Use Only

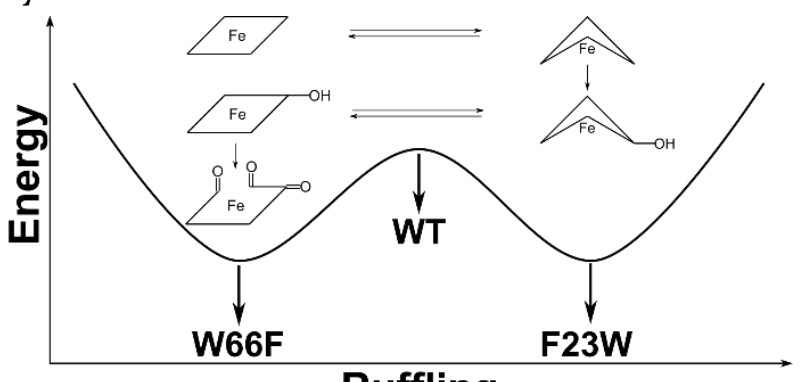

Ruffling 